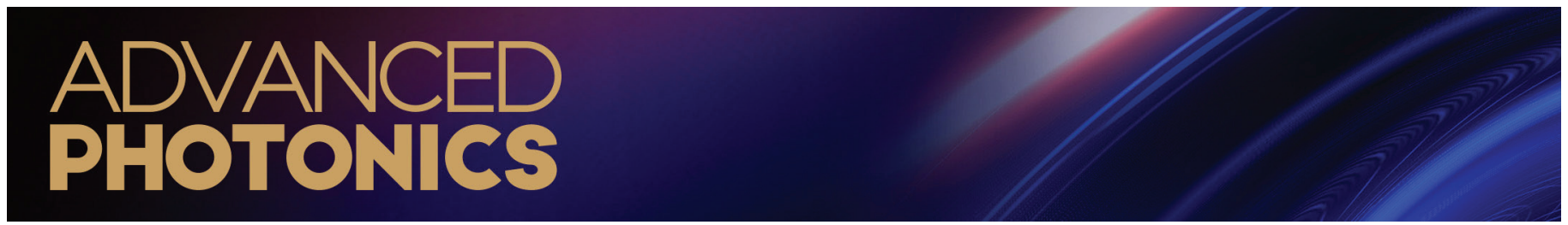

\title{
Preference of subpicosecond laser pulses for terahertz wave generation from liquids
}

\author{
Qi Jin, Yiwen E, Shenghan Gao, and Xi-Cheng Zhang* \\ University of Rochester, The Institute of Optics, Rochester, New York, United States
}

\begin{abstract}
Terahertz ( $\mathrm{THz}$ ) wave generation from laser-induced air plasma generally requires a short temporal laser pulse. In contrast, it was observed that $\mathrm{THz}$ radiation from ionized liquid water prefers a longer pulse, wherein the mechanism remains unclear. We attribute the preference for longer pulse duration to the process of ionization and plasma formation in water, which is supported by a numerical simulation result showing that the highest electron density is achieved with a subpicosecond pulse. The explanation is further verified by the coincidence of our experimental result and simulation when the thickness of the water is varied. Other liquids are also tested to assure the preference for such a pulse is not exclusive to water.
\end{abstract}

Keywords: terahertz wave generation from liquids; laser-induced ionization; plasma.

Received Dec. 3, 2019; accepted for publication Feb. 10, 2020; published online Feb. 27, 2020.

(C) The Authors. Published by SPIE and CLP under a Creative Commons Attribution 4.0 Unported License. Distribution or reproduction of this work in whole or in part requires full attribution of the original publication, including its DOI.

[DOI: 10.1117/1.AP.2.1.015001]

\section{Introduction}

Tremendous interest in terahertz (THz) science and technology has arisen, due to wide applications in nondestructive evaluation, ${ }^{1,2}$ the pharmaceutical industry, ${ }^{3,4}$ nonlinear interaction, ${ }^{5,6}$ and electron acceleration. ${ }^{7,8}$ With successive development, numerous groups have demonstrated $\mathrm{THz}$ wave generation ${ }^{9}$ from solids, ${ }^{10-19}$ gases, ${ }^{20}$ and plasmas. ${ }^{21,22}$ Recently, THz wave generation from liquids under the excitation of intense laser pulses has been reported as well. ${ }^{23-27}$

The generation process of $\mathrm{THz}$ radiation from liquids ${ }^{23,25,26}$ resembles that from gases, due to the fact that laser-induced ionization plays a significant role in both generation processes. In both cases, a model based on a ponderomotive force-induced dipole has been commonly used for the one-color excitation scheme, ${ }^{25,27-29}$ and a transient current model was successfully applied for the two-color excitation scheme. ${ }^{26,30-32}$ Remarkably, one distinction between the two cases is that the dependence of $\mathrm{THz}$ radiation on the optical pulse duration is very different: $\mathrm{THz}$ radiation from gases favors a short optical pulse, whereas a longer pulse offers stronger $\mathrm{THz}$ emission in liquids. The phenomenon was experimentally observed while a physical explanation was still absent. ${ }^{23}$ Moreover, it was observed that only a 10-times enhanced $\mathrm{THz}$ field was obtained when an asymmetric excitation was applied to replace the one-color excitation

*Address all correspondence to Xi-Cheng Zhang, E-mail: xi-cheng.zhang@ rochester.edu scheme in the liquid. ${ }^{26}$ Compared with the many orders of magnitude improvement in the gas, ${ }^{9,22}$ this lower enhancement may be caused by the limitation in optical pulse duration. Studying the effect of pulse duration on the $\mathrm{THz}$ radiation is crucial to understand the involved physics and therefore provides insights to boost $\mathrm{THz}$ emission from liquids.

\section{Experimental Setup}

For the experimental study, liquid lines are introduced as the THz sources. ${ }^{27}$ Figure 1(a) shows a photo of a $260-\mu \mathrm{m}$ diameter water line, which is produced by a $260-\mu \mathrm{m}$ inner diameter syringe needle (BSTEAN ${ }^{\mathrm{TM}}$ ). A Masterflex L/S digital pump from Cole-Parmer ${ }^{\circledR}$ is used to drive the liquid. We define that the liquid line flows along the $y$ direction. A femtosecond amplified laser (Ti:sapphire) with a central wavelength of $800 \mathrm{~nm}$ and a repetition rate of $1 \mathrm{kHz}$ is used for the excitation. The flow velocity of the liquid is controlled to be $7 \mathrm{~m} / \mathrm{s}$. In this case, each laser pulse will interact with a fresh liquid spot. A horizontally polarized laser beam with $0.4 \mathrm{~mJ}$ pulse energy propagating along the $z$ direction is focused into the liquid by a 2 -in. effective focal length lens $(F / 4)$ to generate $\mathrm{THz}$ waves. A highresistivity silicon wafer acts as a filter to block the residual laser beam while allowing the $\mathrm{THz}$ beam to pass through. The $\mathrm{THz}$ electric field is measured by a $2-\mathrm{mm}$ thick, $\langle 110\rangle$-cut ZnTe crystal placed in the direction of laser propagation through electrooptic sampling. ${ }^{33}$ 


\section{Results of Experiments and Simulations}

\subsection{THz Fields from a Water Line}

Figure 1(b) shows the peak values of $\mathrm{THz}$ fields when the $260-\mu \mathrm{m}$ diameter water line is scanned along the $x$ direction across the laser focal point. For $x= \pm 150 \mu \mathrm{m}$, the signal from water is also recorded even though the laser focal point is in the air. The ionization in the water still occurs when the focus is not far away from the water surface. A weak THz signal is detected at $x=0 \mu \mathrm{m}$, which is represented as the black dot in the middle. This coincides with the case of a water film with a normally incident laser beam. ${ }^{25}$ In contrast, the $\mathrm{THz}$ signal becomes significant when the water line is shifted away from the zero position in the $x$ direction. The $\mathrm{THz}$ field is maximized at $x= \pm 90 \mu \mathrm{m}$, which is caused by the ponderomotive force-induced current with the symmetry broken around the interface. ${ }^{27}$ The value of the $\mathrm{THz}$ peak field changes in sign from negative (red dots) to positive (blue dots) when the $x$ position varies from negative to positive. If two points are symmetric about 0 , their absolute values of $\mathrm{THz}$ peak field are identical. The waveforms of the $\mathrm{THz}$ signals at $x= \pm 90 \mu \mathrm{m}$ are shown in Fig. 1(c). Clearly, the THz waveforms flip over when their $x$ positions are mirrored with respect to the zero position. The results shown in Figs. 1(b) and 1(c) can also be explained by the dipole model: ${ }^{25}$ mirrored $x$ position leads to an opposite projected direction of the dipole in water, resulting in an inverted $\mathrm{THz}$ waveform. For $x=0 \mu \mathrm{m}$, the dipole in water is oriented along the direction of laser propagation contributing to weak $\mathrm{THz}$ radiation in the $z$ direction. Thus a tilted dipole realized by a shift of the water line in the $x$ direction is essential to obtain the strongest $\mathrm{THz}$ signal in the direction of laser propagation. In the following sections, $x=90 \mu \mathrm{m}$ will be used for the experiment.

\subsection{Optimal Pulse Duration}

To explore the dependence of $\mathrm{THz}$ radiation on the optical pulse duration, laser pulses are stretched in time by adjusting the compressor stage integrated within the laser to achieve various pulse durations while the laser pulse energy is kept the same. The corresponding $\mathrm{THz}$ energy from a $210-\mu \mathrm{m}$ diameter water line is recorded and shown as the black dot in Fig. 2. Unlike the case of air, a subpicosecond pulse (345 fs) is required for the

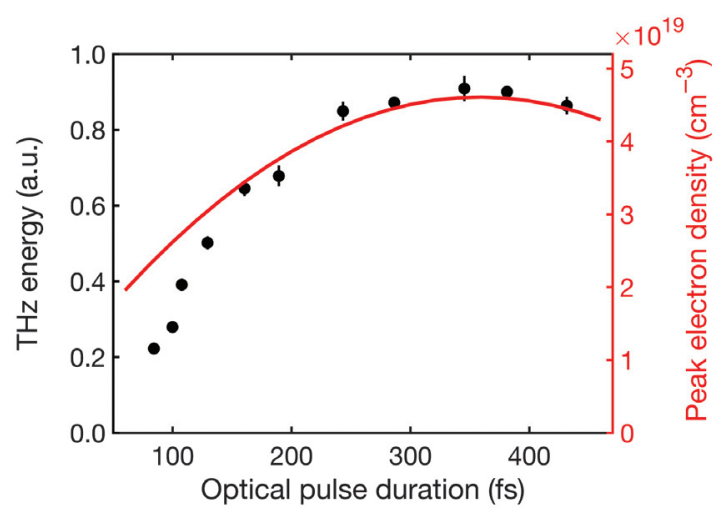

Fig. 2 Effect of optical pulse duration on THz energy and peak electron density for a $210-\mu \mathrm{m}$ water line. The black dots are the experimental data for $\mathrm{THz}$ energy. The red curve is the simulation data for peak electron density.

optimization of $\mathrm{THz}$ radiation from liquid water. In this paper, subpicosecond is defined as 200 to $800 \mathrm{fs}$.

The result of the optimal pulse duration can be understood from the interaction between the laser field $E(z, t)$ and the water, which is described by the following wave equation: ${ }^{34}$

$$
\begin{aligned}
\frac{\partial E}{\partial z}= & \frac{i}{2 n k_{0}} \nabla_{T}^{2} E+i k_{0} n_{2}|E|^{2} E-\frac{i \beta_{2}}{2} \frac{\partial^{2} E}{\partial t^{2}} \\
& -\frac{\sigma}{2}(1+i \omega \tau) \rho E-\frac{\beta^{(K)}}{2}|E|^{2 K-2} E
\end{aligned}
$$

where the terms on the right-hand side represent transverse beam diffraction, self-focusing (SF), group velocity dispersion (GVD), plasma absorption and defocusing, and multiphoton absorption, respectively. Here $n$ is the refractive index, $k_{0}$ is the wave vector, $n_{2}$ is the nonlinear coefficient, $\beta_{2}=\partial^{2} k / \partial \omega^{2}$ represents GVD, $\sigma=\left(k e^{2} \tau / m \omega \epsilon_{0}\right) /\left(1+\omega^{2} \tau^{2}\right)$ is the cross section for inverse Bremsstrahlung absorption, ${ }^{34,35} \omega$ is the optical frequency, $\tau$ is the electron collision time, $\rho$ is the electron density, and $\beta^{(K)}$ is the nonlinear coefficient for $K$-photon absorption.

Electrons are produced by intense laser pulses through multiphoton ionization (MPI)/tunneling ionization (TI) and cascade ionization at the focus. ${ }^{34,36-40}$ MPI/TI directly ionizes water (a)

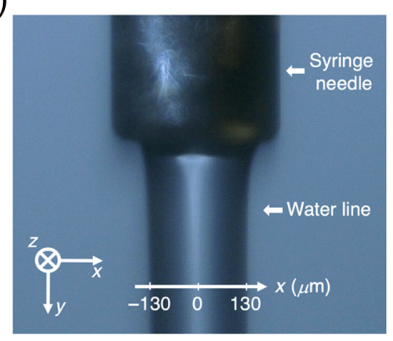

(b)

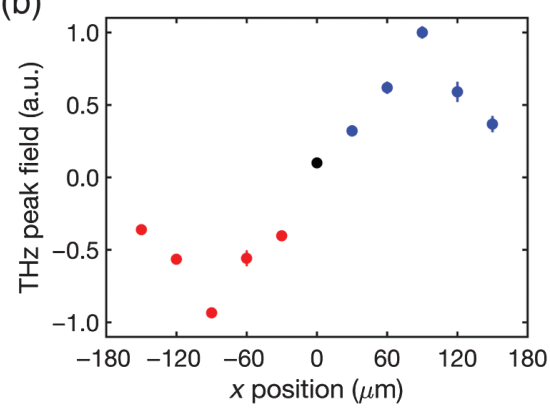

(c)

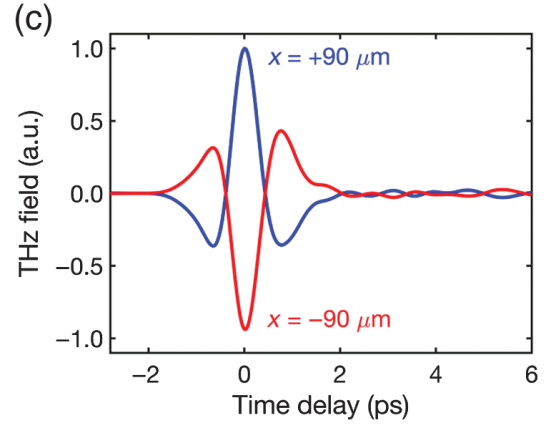

Fig. 1 (a) Photograph of the water line produced by a syringe needle in a side view. The diameter of the water line is $260 \mu \mathrm{m}$. Its flowing velocity is $7 \mathrm{~m} / \mathrm{s}$ along the $y$ direction. The laser beam propagates in the $z$ direction. The water line can be moved along the $x$ direction by a translation stage. (b) THz peak fields with different $x$ positions when the $260-\mu \mathrm{m}$ diameter water line is crossing the laser focal point along the $x$ direction. (c) THz waveforms at $x= \pm 90 \mu \mathrm{m}$ in (b). 
molecules while cascade ionization desires the presence of free electrons for initiation. These free electrons come from the background electrons and MPI/TI. In our experimental condition, the laser intensity at the focus is at the level of $10^{15} \mathrm{~W} / \mathrm{cm}^{2}$. The Keldysh parameter $\gamma=0.23$ is obtained when water is treated as an amorphous semiconductor with a bandgap of $U=6.5 \mathrm{eV},{ }^{36,41-43}$ indicating that TI dominates over MPI in our case..$^{40,44}$ Thus the last term of Eq. (1) needs to be modified to $\frac{\eta_{\mathrm{TI}}\left(\rho_{\text {water }}-\rho\right) U}{2 I} E$ when TI dominates. ${ }^{45}$ The electron density $\rho(z, t)$ satisfies the rate equation:

$\frac{\partial \rho}{\partial t}=\eta_{\mathrm{TI}}\left(\rho_{\text {water }}-\rho\right)+\eta_{\text {cas }} \rho-\eta_{\text {diff }} \rho-\eta_{\text {rec }} \rho^{2}$.

The first two terms on the right-hand side correspond to the generation of electrons from TI and cascade ionization. The other two terms describe the loss of electrons from diffusion and recombination. $\rho_{\text {water }}$ is the molecular density of water. The laser pulse is considered to have a Gaussian profile with its intensity as follows:

$I(t)=0.94 \frac{\varepsilon_{p}}{\tau_{p}} \exp \left[-4 \ln 2\left(\frac{t}{\tau_{p}}\right)^{2}\right] /\left(0.5 \pi w_{0}^{2}\right)$,

where $\varepsilon_{p}$ is the laser pulse energy, $\tau_{p}$ is the laser pulse duration, and $w_{0}$ is the beam waist at the focus.

It is worth underlining that each cascade process needs an ionization time $\tau_{\text {ion }}$ to perform. Thus one seed electron cannot produce more than $2^{\tau_{p}} / \tau_{\text {ion }}$ electrons through the cascade process, regardless of the laser intensity. Comparing with the increase of electrons due to TI, the exponential boost caused by cascade ionization makes it the dominating ionization process in water when $\tau_{p}$ is above $40 \mathrm{fs} .{ }^{37}$ Therefore, a longer pulse duration that allows for more cascades taking place benefits plasma formation through the exponential increase from cascade ionization. Even though the majority of electrons are provided by cascade ionization, TI is still quite important because it provides most of the electrons to initiate the cascade process, especially in pure water. Since TI highly depends on laser intensity and laser intensity is inversely proportional to $\tau_{p}$ if the laser pulse energy $\varepsilon_{p}$ is fixed [see Eq. (3)], generated electron density will start to decrease if $\tau_{p}$ raises too much. Considering the fact that higher electron density contributes to more $\mathrm{THz}$ radiation, the trade-off between the two effects can lead to the preference for a subpicosecond pulse.

Simulations are carried out to verify the theoretical analysis. The laser wavelength and pulse energy are set to match the values in the experiment. In our experiment, a strong external focusing is used, where the SF and the geometrical focusing become indistinguishable. ${ }^{46}$ Thus the term of SF is not included in the simulation. We also assume that the impact of GVD is insignificant ${ }^{47}$ because the plasma length of our interest is short ( $\sim 200 \mu \mathrm{m})$. The electron collision time $\tau=1$ fs and the cascade ionization time $\tau_{\text {ion }}=5 \mathrm{fs}$ are applied. ${ }^{37}$ Since femtosecond and subpicosecond laser pulses are used in the experiment, contribution from diffusion is justifiably neglected. ${ }^{34,37}$ The TI rate $\eta_{\mathrm{TI}}(t)$ can be acquired by the ADK model. ${ }^{48}$ The cascade ionization rate $\eta_{\text {cas }}$ is calculated as in Ref. 37 . The recombination rate $\eta_{\text {rec }}$ is taken to be $2 \times 10^{-9} \mathrm{~cm}^{3} / \mathrm{s}^{37,49}$ The molecular density of water $\rho_{\text {water }}$ is $3.34 \times 10^{22} \mathrm{~cm}^{-3} .^{36}$ The initial electron density is chosen to be $\rho_{0}=10^{10} \mathrm{~cm}^{-3}$, which will hardly impact on the evolution of electron density even if $\rho_{0}$ is changed over several orders of magnitude. ${ }^{34,50}$ By numerically solving ${ }^{34}$ the coupled Eqs. (1) and (2) with different values of pulse duration, the evolution of electron density $\rho(z, t)$ that corresponds to different $\tau_{p}$ is obtained. Consequently, the peak electron density versus $\tau_{p}$ is achieved and plotted (see the red curve in Fig. 2). As expected, a subpicosecond pulse is a requisite for the highest peak electron density.

For further verification, the diameter of the water line $d$ is chosen as a variable to see how the optimal pulse duration changes with the water line diameter in the experiment and simulation. Experimentally, seven individual syringe needles with different inner diameters are used to produce water lines with diameters varying from tens of microns to half a millimeter. The optimal pulse duration for the $\mathrm{THz}$ energy from each water line is recorded and shown as the red dot in Fig. 3. The optimal pulse duration gradually changes from 257 to 513 fs when $d$ increases from 90 to $510 \mu \mathrm{m}$. In the simulation, the evolution of electron density that corresponds to different $\tau_{p}$ is achieved. Then the peak electron density is integrated over $z$ within the whole range of the water line that is determined by the diameter $d$. Thus the optimal pulse duration for the highest electron density that corresponds to the different diameter of the water line is obtained (see the blue squares in Fig. 3). To make the result clear to see, only a few points in a similar range of the experiment are shown. The coincidence of the experimental result and the simulation supports our model and explanation.

\subsection{Applicability to Other Liquids}

To confirm that the preference for a subpicosecond laser pulse is not exclusive to water, other liquids such as $\alpha$-pinene, $\mathrm{p}$-xylene, and ethanol are experimentally tested as well. It is noticeable that there are some material-related parameters involved in the simulations, such as refractive index, nonlinear coefficient, ionization energy, ionization time, and molecular density. These parameters may take effect as well. In our experiment, all these liquids exhibit an optimal pulse duration around $345 \mathrm{fs}$ when their diameters have the same value of $210 \mu \mathrm{m}$. Therefore, these parameters do not substantially affect the optimal pulse duration. However, they still demonstrate the influence on the $\mathrm{THz}$ radiation. Here we use $\alpha$-pinene as an example to show

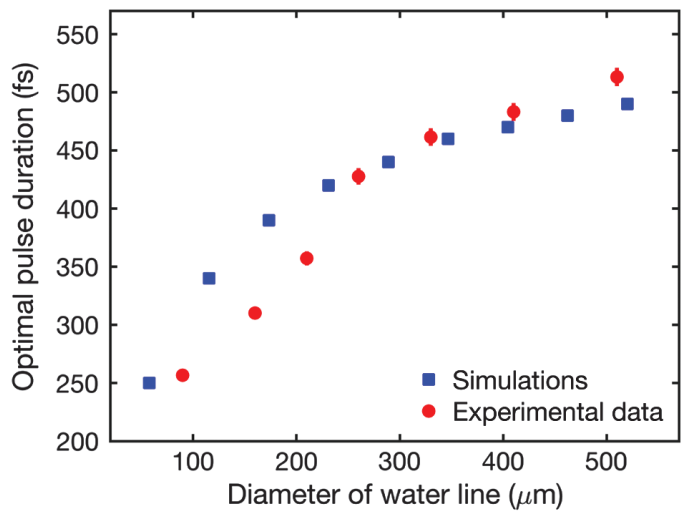

Fig. 3 Optimal optical pulse duration versus the diameter of the water line. The blue squares are simulations of optimal pulse duration aiming for highest electron density. The red dots are the experimental data obtained with strongest $\mathrm{THz}$ energy. 
(a)
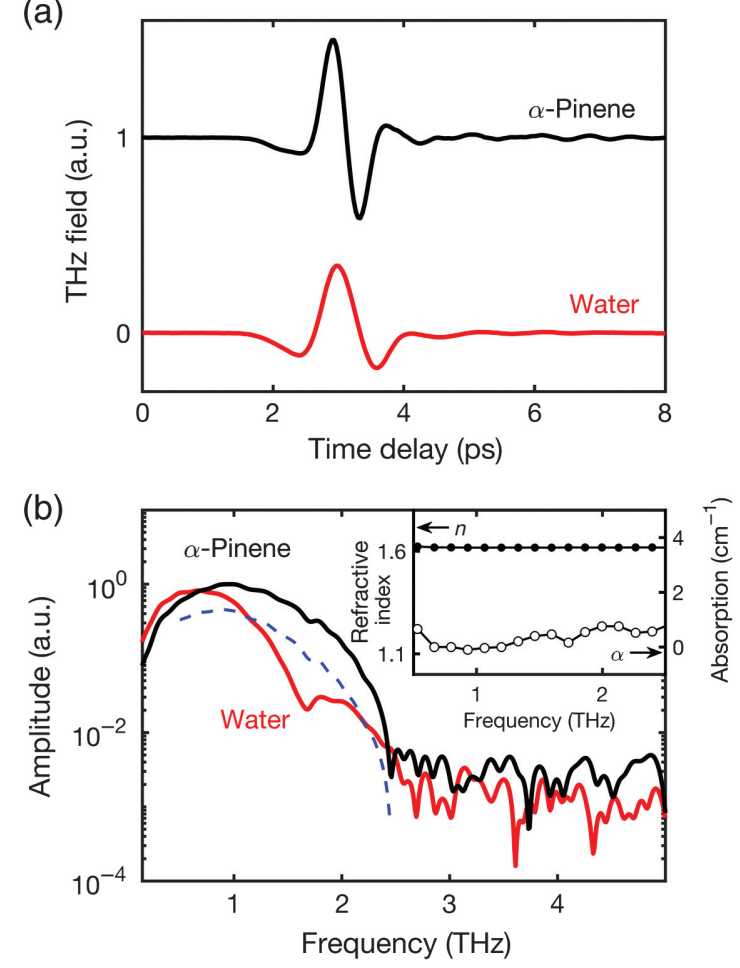

Fig. 4 Comparison of THz radiation generated from $\alpha$-pinene and water in (a) time domain and (b) frequency domain. Optical pulse durations are individually optimized for $\alpha$-pinene and water. They have the same value of $345 \mathrm{fs}$. The diameter of the liquid line is $210 \mu \mathrm{m}$. Laser pulse energy is $0.4 \mathrm{~mJ}$. The dash line in (b) is calculated by removing the absorption of $\alpha$-pinene and adding the absorption of water to the black curve from 0.5 to $2.5 \mathrm{THz}$. Inset: measured results of refractive index $n$ (dots) and field absorption coefficient $\alpha$ (circles) of $\alpha$-pinene within 0.5 to $2.5 \mathrm{THz}$.

its comparison with water. Figure 4(a) shows the $\mathrm{THz}$ waveforms that are generated from a $210-\mu \mathrm{m}$ diameter $\alpha$-pinene line and a $210-\mu \mathrm{m}$ diameter water line. The $\alpha$-pinene emits a stronger $\mathrm{THz}$ field, which is about 1.8-times stronger than that from the water in the same experimental condition. In the frequency domain, as shown in Fig. 4(b), the $\alpha$-pinene offers more high-frequency components and provides a wider bandwidth.

To understand the difference between the signals from water and $\alpha$-pinene, the refractive index and field absorption coefficient of $\alpha$-pinene are measured by a standard THz-time domain spectroscopy system. ${ }^{51}$ The results are shown in the inset of Fig. 4(b). $\alpha$-pinene has a refractive index $n$ of 1.62 from 0.5 to $2.5 \mathrm{THz}$ with little dispersion. Its absorption in such a frequency range is very small $\left(\alpha<2 \mathrm{~cm}^{-1}\right)$. The strong absorption of water is not the only contributor to the difference between $\alpha$-pinene and water. To confirm this, the absorption from $\alpha$ pinene is removed from the experimental data (black curve) and the absorption from water is added..$^{52}$ This treatment of ignoring the change of absorption caused by the plasma formation is justified because only $0.2 \%$ of molecules are ionized in our case. The influence of their different refractive indices on the Fresnel coefficients of the interfaces is included. The result is plotted as the blue dash line in Fig. 4(b). The discrepancy between the blue dash line and the red curve reveals that there are other contributors involved. The material-related parameters function in the ionization process and thus may affect the $\mathrm{THz}$ radiation resulting in the observation in Fig. 4. In this paper, we mainly focus on the process of plasma formation for the $\mathrm{THz}$ emission. Further investigation into $\mathrm{THz}$ radiation from plasma current ${ }^{25,27,53-55}$ will be needed to fully understand the result shown in Fig. 4.

\subsection{Discussion}

We note that the subpicosecond optimal pulse duration for $\mathrm{THz}$ wave generation was also previously reported by Wang et al. ${ }^{56}$ The preference for longer pulses was attributed to the longer interaction time of the gas atoms with the laser. Even though the result has similarities to ours, they did study a different case, which is the two-color excitation scheme in gases, whereas our work focuses on the one-color case in liquids.

It also needs to be mentioned that the demonstration of preference for a subpicosecond optical pulse can also be used to explain the low enhancement of $\mathrm{THz}$ radiation under the twocolor excitation. ${ }^{26}$ The subpicosecond pulse is indispensable to achieve high electron density in the liquid, as we have just demonstrated. Nevertheless, a short pulse works much better for the second-harmonic generation from the fundamental beam. The discrepant demand regarding the pulse duration significantly limits the generation of $\mathrm{THz}$ waves under the two-color excitation. Considering that the $\mathrm{THz}$ field strength of $0.2 \mathrm{MV} / \mathrm{cm}$ from the water was claimed by Zhang et al. ${ }^{27}$ under the one-color excitation, a very intense $\mathrm{THz}$ radiation could be expected if the requirements for pulse duration are satisfied under the two-color excitation. One possible solution is separately controlling the phase and polarization of the fundamental beam and the second-harmonic beam using a dichroic mirror. ${ }^{57}$ Another dichroic mirror can be applied to combine the second-harmonic beam and the stretched fundamental beam. Such an arrangement assures that the plasma would be generated by a time-stretched pulse and that the second-harmonic component remains intense.

\section{Conclusion}

To summarize, the preference for a subpicosecond laser pulse for $\mathrm{THz}$ wave generation from liquids is understood from the process of laser-induced plasma formation based on our experimental results and simulations. On one hand, cascade ionization, the dominant ionization process leading to an exponential increase in the number of electrons, needs a long pulse duration. On the other hand, increasing the pulse duration sacrifices the laser intensity, which limits the generation of plasma in the liquid. The trade-off between the two effects results in an optimal pulse duration around the subpicosecond region. Unlike the speculation given in Ref. 23 where cascade ionization is the only reason for the preference, electron collision time and TI are demonstrated to be critical factors as well. Other liquids such as $\alpha$-pinene, $\mathrm{p}$-xylene, and ethanol are also tested to ensure that the preference for a subpicosecond pulse does not only occur in water. In addition to unveiling the influence of optical pulse duration on laser-induced plasma formation for $\mathrm{THz}$ radiation, our observations also contribute insight into the development of intense liquid $\mathrm{THz}$ sources.

\section{Acknowledgments}

This work was supported by the U.S. National Science Foundation (No. ECCS-1916068), the Army Research Office 
(No. W911NF-17-1-0428), and the Air Force Office of Scientific Research (No. FA9550-18-1-0357).

\section{References}

1. K. Kawase et al., "Non-destructive terahertz imaging of illicit drugs using spectral fingerprints," Opt. Express 11(20), 25492554 (2003).

2. N. Karpowicz et al., "Compact continuous-wave subterahertz system for inspection applications," Appl. Phys. Lett. 86(5), 054105 (2005).

3. B. Ferguson and X.-C. Zhang, "Materials for terahertz science and technology," Nat. Mater. 1(1), 26-33 (2002).

4. L. Ho, M. Pepper, and P. Taday, "Terahertz spectroscopy: signatures and fingerprints," Nat. Photonics 2(9), 541-543 (2008).

5. Y. Shen et al., "Nonlinear cross-phase modulation with intense single-cycle terahertz pulses," Phys. Rev. Lett. 99(4), 043901 (2007).

6. D. Turchinovich, J. M. Hvam, and M. C. Hoffmann, "Self-phase modulation of a single-cycle terahertz pulse by nonlinear free-carrier response in a semiconductor," Phys. Rev. B 85(20), 201304 (2012).

7. E. A. Nanni et al., "Terahertz-driven linear electron acceleration," Nat. Commun. 6, 8486 (2015).

8. D. Zhang et al., "Segmented terahertz electron accelerator and manipulator (steam)," Nat. Photonics 12(6), 336-342 (2018).

9. X.-C. Zhang, A. Shkurinov, and Y. Zhang, "Extreme terahertz science," Nat. Photonics 11(1), 16-18 (2017).

10. F. Blanchard et al., "Generation of intense terahertz radiation via optical methods," IEEE J. Sel. Top. Quantum Electron. 17(1), 5-16 (2011).

11. K.-L. Yeh et al., "Generation of $10 \mu \mathrm{J}$ ultrashort terahertz pulses by optical rectification," Appl. Phys. Lett. 90(17), 171121 (2007).

12. X.-C. Zhang et al., "Generation of femtosecond electromagnetic pulses from semiconductor surfaces," Appl. Phys. Lett. 56(11), 1011-1013 (1990).

13. M. Van Exter, C. Fattinger, and D. Grischkowsky, "High-brightness terahertz beams characterized with an ultrafast detector," Appl. Phys. Lett. 55(4), 337-339 (1989).

14. X.-C. Zhang and D. Auston, "Optoelectronic measurement of semiconductor surfaces and interfaces with femtosecond optics," J. Appl. Phys. 71(1), 326-338 (1992).

15. F. Kadlec, P. Kužel, and J.-L. Coutaz, "Optical rectification at metal surfaces," Opt. Lett. 29(22), 2674-2676 (2004).

16. G. H. Welsh, N. T. Hunt, and K. Wynne, "Terahertz-pulse emission through laser excitation of surface plasmons in a metal grating," Phys. Rev. Lett. 98(2), 026803 (2007).

17. J. Dai and X.-C. Zhang, "Terahertz wave generation from thin metal films excited by asymmetrical optical fields," Opt. Lett. 39(4), 777-780 (2014).

18. A. Sagisaka et al., "Simultaneous generation of a proton beam and terahertz radiation in high-intensity laser and thin-foil interaction," Appl. Phys. B 90(3-4), 373-377 (2008).

19. G. Liao et al., "Multimillijoule coherent terahertz bursts from picosecond laser-irradiated metal foils," Proc. Natl. Acad. Sci. U. S. A. 116(10), 3994-3999 (2019).

20. M. S. Tobin, "A review of optically pumped NMMW lasers," Proc. IEEE 73(1), 61-85 (1985).

21. T. Löffler, F. Jacob, and H. Roskos, "Generation of terahertz pulses by photoionization of electrically biased air," Appl. Phys. Lett. 77(3), 453-455 (2000).

22. D. Cook and R. Hochstrasser, "Intense terahertz pulses by fourwave rectification in air," Opt. Lett. 25(16), 1210-1212 (2000).

23. Q. Jin et al., "Observation of broadband terahertz wave generation from liquid water," Appl. Phys. Lett. 111(7), 071103 (2017).

24. I. Dey et al., "Highly efficient broadband terahertz generation from ultrashort laser filamentation in liquids," Nat. Commun. 8(1), 1184 (2017).
25. Y. E et al., "Terahertz wave generation from liquid water films via laser-induced breakdown," Appl. Phys. Lett. 113, 181103 (2018).

26. Q. Jin et al., "Terahertz wave emission from a liquid water film under the excitation of asymmetric optical fields," Appl. Phys. Lett. 113(26), 261101 (2018).

27. L.-L. Zhang et al., "Strong terahertz radiation from a liquid-water line," Phys. Rev. Appl. 12(1), 014005 (2019).

28. H. Hamster et al., "Subpicosecond, electromagnetic pulses from intense laser-plasma interaction," Phys. Rev. Lett. 71(17), 2725-2728 (1993).

29. H. Hamster et al., "Short-pulse terahertz radiation from high-intensity-laser-produced plasmas," Phys. Rev. E 49(1), 671-677 (1994).

30. K.-Y. Kim et al., "Terahertz emission from ultrafast ionizing air in symmetry-broken laser fields," Opt. Express 15(8), 4577-4584 (2007).

31. K.-Y. Kim et al., "Coherent control of terahertz supercontinuum generation in ultrafast laser-gas interactions," Nat. Photonics 2(10), 605-609 (2008).

32. K.-Y. Kim, "Generation of coherent terahertz radiation in ultrafast laser-gas interactions," Phys. Plasmas 16(5), 056706 (2009).

33. Q. Wu and X.-C. Zhang, "Free-space electro-optic sampling of terahertz beams," Appl. Phys. Lett. 67(24), 3523-3525 (1995).

34. Q. Feng et al., "Theory and simulation on the threshold of water breakdown induced by focused ultrashort laser pulses," IEEE J. Quantum Electron. 33(2), 127-137 (1997).

35. M. Feit and J. Fleck Jr., "Effect of refraction on spot-size dependence of laser-induced breakdown," Appl. Phys. Lett. 24(4), 169172 (1974)

36. P. K. Kennedy, "A first-order model for computation of laserinduced breakdown thresholds in ocular and aqueous media. I. Theory," IEEE J. Quantum Electron. 31(12), 2241-2249 (1995).

37. J. Noack and A. Vogel, "Laser-induced plasma formation in water at nanosecond to femtosecond time scales: calculation of thresholds, absorption coefficients, and energy density," IEEE $J$. Quantum Electron. 35(8), 1156-1167 (1999).

38. C. DeMichelis, "Laser induced gas breakdown: a bibliographical review," IEEE J. Ouantum Electron. 5(4), 188-202 (1969).

39. Y.-R. Shen, The Principles of Nonlinear Optics, p. 575, WileyInterscience, New York (1984).

40. L. Keldysh et al., "Ionization in the field of a strong electromagnetic wave," Sov. Phys. JETP 20(5), 1307-1314 (1965).

41. P. K. Kennedy, D. X. Hammer, and B. A. Rockwell, "Laserinduced breakdown in aqueous media," Prog. Quantum Electron. 21(3), 155-248 (1997).

42. F. Williams, S. Varma, and S. Hillenius, "Liquid water as a lonepair amorphous semiconductor," J. Chem. Phys. 64(4), 1549-1554 (1976).

43. C. Sacchi, "Laser-induced electric breakdown in water," J. Opt. Soc. Am. B 8(2), 337-345 (1991).

44. S. Chin, "From multiphoton to tunnel ionization," in Advances in Multi-Photon Processes and Spectroscopy, S. H. Lin, A. A. Villaeys, and Y. Fujimura, Eds., Vol. 16, pp. 249-271, World Scientific Group (2004).

45. A. Couairon and A. Mysyrowicz, "Femtosecond filamentation in transparent media," Phys. Rep. 441(2-4), 47-189 (2007).

46. S. L. Chin, Femtosecond Laser Filamentation, Vol. 55, Springer, New York (2010).

47. C. Milián et al., "Effect of input pulse chirp on nonlinear energy deposition and plasma excitation in water," J. Opt. Soc. Am. B 31(11), 2829-2837 (2014).

48. M. Ammosov et al., "Tunnel ionization of complex atoms and of atomic ions in an alternating electric field," Sov. Phys. JETP 64(6), 1191 (1986).

49. F. Docchio, "Lifetimes of plasmas induced in liquids and ocular media by single Nd:YAG laser pulses of different duration," Europhys. Lett. 6(5), 407-412 (1988). 
50. Q. Feng et al., "Laser-induced breakdown versus self-focusing for focused picosecond pulses in water," Opt. Lett. 20(19), 1958-1960 (1995).

51. T. D. Dorney, R. G. Baraniuk, and D. M. Mittleman, "Material parameter estimation with terahertz time-domain spectroscopy," J. Opt. Soc. Am. A 18(7), 1562-1571 (2001).

52. C. Ro/nne and L. Thrane, "Investigation of the temperature dependence of dielectric relaxation in liquid water by $\mathrm{THz}$ reflection spectroscopy and molecular dynamics simulation," J. Chem. Phys. 107(14), 5319-5331 (1997).

53. I. Babushkin et al., "Ultrafast spatiotemporal dynamics of terahertz generation by ionizing two-color femtosecond pulses in gases," Phys. Rev. Lett. 105(5), 053903 (2010).

54. L. Bergé et al., "3D numerical simulations of thz generation by two-color laser filaments," Phys. Rev. Lett. 110(7), 073901 (2013).

55. P. Sprangle et al., "Ultrashort laser pulses and electromagnetic pulse generation in air and on dielectric surfaces," Phys. Rev. E 69(6), 066415 (2004).

56. W.-M. Wang et al., "Strong, tunable terahertz emission by twocolor picosecond laser irradiation," Phys. Rev. A 90(2), 023808 (2014).

57. J. Dai and X.-C. Zhang, "Terahertz wave generation from gas plasma using a phase compensator with attosecond phase-control accuracy," Appl. Phys. Lett. 94(2), 021117 (2009).

Qi Jin is an optical engineer at Xerox Corporation. With a BE from Huazhong University of Science and Technology in 2012, he received his MS (2017) and will earn a PhD (2020) in optics from the University of Rochester. He was awarded the James Fienup Academic Achievement Award (2016), the IRMMW-THz Outstanding Student Paper Award
(2017), and the Rochester Precision Optics Graduate Project Award (2018).

Yiwen E is a postdoctoral associate of The Institute of Optics, University of Rochester, NY. She received her PhD in optics from University of the Chinese Academy of Sciences in 2017. Her main research interests are terahertz wave generation and detection, terahertz wave-matter interaction, and ultrafast optics. Currently, she focuses on the project of terahertz wave generation from ionized liquids, especially from liquid water.

Shenghan Gao is a PhD student at University of Pittsburgh. He received his master's degree in optics from University of Rochester; there, he joined Professor Xi-Cheng Zhang's Terahertz Research Group and finished his thesis in liquids generating $\mathrm{THz}$ area. He also earned his bachelor's degree in optics engineering and applied mathematics at University of Rochester.

Xi-Cheng Zhang is the Parker Givens Chair and former director of The Institute of Optics, University of Rochester (UR), NY. Prior to joining UR, he pioneered research in the field of ultrafast laser-based terahertz technology and optical physics at Rensselaer Polytechnic Institute (RPI), Troy, NY (1992-2012). At RPI, he was founding director of the Center for $\mathrm{THz}$ Research. With a BS (1982) from Peking University, he earned the MS (1983) and PhD degree (1986) in physics from Brown University, Rhode Island. He has been recognized by many honors and awards, including the Humboldt Prize, from the Alexander von Humboldt Foundation (2018); IRMMW-THz Kenneth Button Prize (2014); OSA William F. Meggers Award (2012); IEEE Photonics Society William Streifer Scientific Achievement Award (2011); and the Rensselaer William H. Wiley 1866 Award (2009), among others. 